\title{
Preoperative Chest Radiographic Examination for Pediatric Inguinal Hernia and Umbilical Hernia
}

\author{
Hirotaka Kato $^{1}{ }^{10} \cdot$ Akio Kubota $^{1} \cdot$ Yasuyuki Mitani $^{1} \cdot$ Taro Goda $^{1} \cdot$ Hiroki Yamaue ${ }^{1}$
}

Received: 31 October 2021 / Accepted: 10 December 2021/Published online: 24 January 2022

(c) Dr. K C Chaudhuri Foundation 2021

To the Editor: Preoperative chest radiographic examination is performed to exclude heart or respiratory disease, and to evaluate the status [1]. Most Japanese pediatric institutions also perform preoperative chest radiographic examination even for inguinal or umbilical hernia. Indication for preoperative chest radiographic examination has been, however, rarely discussed, especially in children without underlying disease related to respiratory and circulatory dynamics, and those without significant physical findings. It was, therefore, investigated in the present study whether a preoperative chest radiographic examination is required for healthy children with inguinal and umbilical hernia.

Six hundred and forty-nine patients who underwent surgery for inguinal or umbilical hernia between January 2005 and December 2020 were enrolled in the present study, excluding 8 patients with very low birth weight and chronic respiratory disease, 8 patients with asthma and undergoing oral or inhalation treatment, 15 patients with history with congenital heart disease, and 13 patients that underwent emergency surgery for inguinal hernia incarceration. The effects of chest radiographic examination on the scheduled surgery and anesthesia methods were retrospectively analyzed.

Preoperative chest radiographic examination showed enlargement of cardiac shadow in 2 patients and decrease of lung-field permeability in 1 patient. As they all showed no significant physical findings, there was no postponement of surgery, change of anesthesia method, or postoperative complications.
Specific guidelines for the indication of preoperative chest radiographic examination do not exist in Japan, although European Society of Anesthesia guidelines for preoperative chest radiographic examination recommended that it should be taken for those with cardiovascular disease, respiratory disease, renal disease, diabetes, obesity, abnormal blood coagulation, or anemia, and the elderly [2].

Preoperative chest radiographic examination in pediatric inguinal or umbilical hernia, especially for children without significant physiological findings or underlying diseaserelated respiratory and circulatory system problems, was thought to be unnecessary.

\section{Declarations \\ Conflict of Interest None.}

\section{References}

1. Garcia-Miguel FJ, Serrano-Aguilar PG, Lopez-Bastida J. Preoperative assessment. Lancet. 2003;362:1749-57.

2. Alsumait BM, Alhumood SA, Ivanova T, Mores M, Edeia M. A prospective evaluation of preoperative screening laboratory tests in general surgery patients. Med Princ Pract. 2002;11:42-5.

Publisher's Note Springer Nature remains neutral with regard to jurisdictional claims in published maps and institutional affiliations.

Hirotaka Kato

hirotaka@wakayama-med.ac.jp

1 Second Department of Surgery, Wakayama Medical University Hospital, 811-1 Kimiidera, Wakayama, Wakayama 641-8509, Japan 